\title{
GALAXY FORMATION AND LARGE-SCALE STRUCTURES IN A TWO-COMPONENT DARK MATTER SCENARIO
}

\author{
Masayuki UMEMURA \\ Tokyo Astronomical Observatory, Mitaka, Tokyo 181, Japan
}

\begin{abstract}
A universe dominated by both hot (HDM) and cold dark matter (CDM) is proposed. In this context, the new features for the formation of dwarf galaxies, Ly $\alpha$ clouds, galaxies, and large-scale structures are presented.
\end{abstract}

Biased CDM models may reproduce the galaxy distributions as observed (White et al. 1987), but the recently reported large-scale streaming motion is seriously challenging the biased models. Recently Peacock et -al. (1987) have shown that the massive neutrino model can yield the required cosmic drift velocities. Nevertheless the pure neutrino model produces so highly peaked features in galaxy distributions, and also is not welcome for the formation of dwarf galaxies and unclustered Ly $\alpha$ clouds. The two-component dark matter scenario (hybrid model) has been designed to remove these defects (Umemura and Ikeuchi 1985 (UI); Ikeuchi and Norman 1987).

\section{OVERVIEWS EXPECTED IN THE HYBRID MODEL}

Numerical calculations have been performed in a subgalactic scale, galactic scale, and supercluster scale within the context of the hybrid model.

\section{A. Subgalactic Objects}

The formation of dwarf galaxies and Ly $\alpha$ clouds was investigated in a universe composed of HDM, $\mathrm{CDM}$, and baryonic matter with a spherically symmetric scheme (UI). The first luminous objects are born within CDM halos, in a large-scale high density region produced by a HDM overdense fluctuation, and they are of dwarf galaxy scales. According to the growth of CDM fluctuations, the less massive/luminous galaxies could be less clustered (Giovanelli 1987). On the other hand, in a large-scale low density region, most of subgalactic scale fluctuations are converted to expanding clouds due to UV flux from QSOs. These numerous unclustered clouds yield Ly $\alpha$ absorptions of QSO light to just the same degree as observed. The cloud expansion leads to the decrease of absorption line number with decreasing $z$.

\section{B. Fragmentation of Pancakes and Galaxy Formation}

HDM overdense fluctuations consequently perform 'pancake' collapse. These pancakes consist of a dissipative gas component, and a collisionless component, i.e., HDM and dwarf galaxies. The fragmentation processes of composite pancakes are numerically simulated by a new 3D scheme; a combination of the smoothed particle method for gas particles and $N$-body scheme for collisionless particles. A result is shown in Fig. 1 for $N_{g}=N_{c}=2000, m_{g}=5 \times 10^{8} M \odot$, and $m_{c}=9.5 \times 10^{9} M \odot$. The pancake fragments into many bound systems having $10^{11-12} M_{\odot}$ in total mass and $10^{10-11} M \odot$ in gaseous component, which would evolve to typical size galaxies. 
C. Galaxies in Voids

In the hybrid model, some galaxies are also expected to be born recently in voids, because CDM fluctuations can collapse in time even in HDM underdense regions. These galaxies could be late-type ones through the tidel field of clusters, and form a less-clustered nonvirialized system. Galaxies in voids can be observed directly as young galaxies (Kirshner et al. 1987), or can be detected by metal absorption lines (Brosch and Gondhalekar 1984).

\section{Large-Scale Structures and Streaming Motion}

Large-scale galaxy distributions are determined by both clustered galaxies from pancake fragmentation and less-clustered ones which originate in HDM underdense regions, so galaxy distributions are not so highly peaked on the whole as in a pure HDM model. I have tentatively calculated the galaxy clustering in the hybrid model, in an external gravitational field which is caused by an adjacent large-scale density enhancement (Fig. 2). It is natural that there form sheet/bar-like superclusters and large-scale vacant regions. Moreover, the adjacent overdense region with $\delta \rho / \rho \sim 1$ and a linear scale of $\sim 100 \mathrm{Mpc}$ can produce the large drift velocity, $\sim 700 \mathrm{~km} \mathrm{~s}^{-1}$.
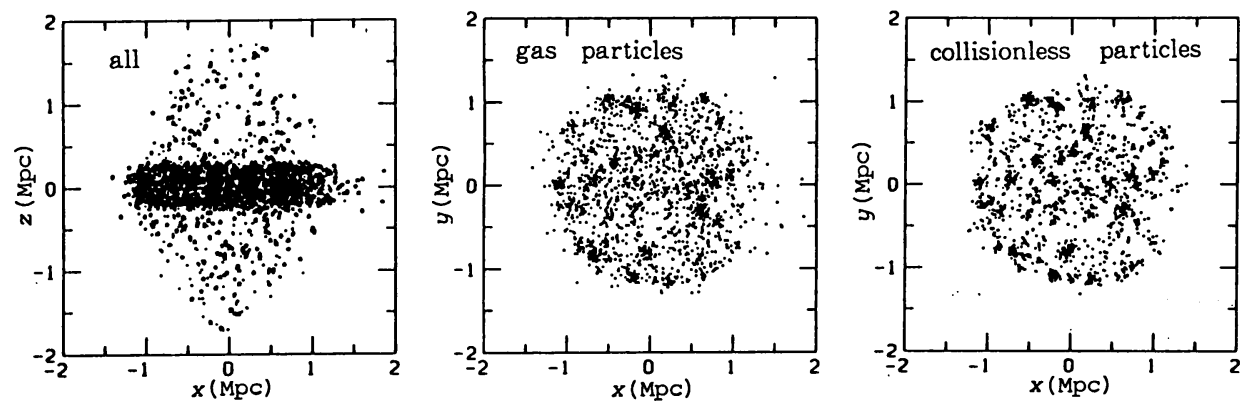

Fig. 1. Pancake fragmentation. The epoch of pancake collapse is $z \sim 4$. The features at $z=1.4$ are polotted.
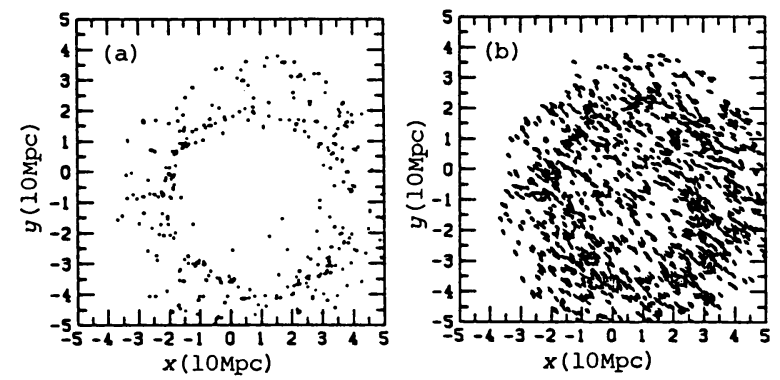

Fig. 2. The galaxy clustering in an external field. The present features are plotted by (a) a slice projection, and (b) a peculiar velocity field of all particles $(N=2000)$.

I am grateful to Dr. Ikeuchi for valuable discussion. This research was supported largely by Inoue Foundation for Science, and in part by the Grants-in-Aid for Encouragement of Young Scientists of the Ministry of Education, Science, and Culture, No. 61790115.

\section{REFERENCES}

Brosch, N, and Gondhalekar, P. M. 1984, Astr. Ap. Letters, 140, 43.

Giovenelli, R. 1987, this volume.

Ikeuchi, S., and Norman, C. A. 1987, Ap. J., 312, 485.

Kirshner, R. P., Oemler, A. Jr., Schechter, P. L., and Shectman, S. A. 1987, Ap. J., 314, 493.

Peacock, J. A, Lumsden, S. L., and Heavens, A. F. 1987, M.N.R.A.S., in press.

Umemura, M, and Ikeuchi, S. 1985, Ap. J, 299, 583.

White, S. D. M., Frenk, C. S., Davis, M., and Efstathiou, G. 1987, Ap. J., 313, 505. 